\title{
Comparative Study between Laparoscopic Assisted and Traditional Vaginal Hysterectomy
}

\author{
Chowdhury L
}

DOI: https://doi.org/10.3329/jafmc.v13i2.41370

\begin{abstract}
Introduction: The choice of hysterectomy approach depends on the reason for hysterectomy, the overall health of the patient and surgeon's experience. The choice of technique dictates hospital stay and healing time.
\end{abstract}

Objective: To compare the efficacy and outcome of Laparoscopic Assisted Vaginal Hysterectomy (LAVH) and traditional Vaginal Hysterectomy (VH).

Materials and Methods: A total of 100 patients were purposively selected for this prospective comparative study during the period of January 2011 to January 2013 in $\mathrm{CMH}$, Dhaka and Hitech Multicare Hospital. Non-malignant patients undergoing $\mathrm{LAVH}$ and $\mathrm{VH}$ by the same surgeon were included in this study. Clinical variables such as operative time, operative cost, blood loss, quantity of analgesics used and hospital stay were observed in both cases for comparison. Several other variables were also observed as postoperative complications such as amount of haemoglobin decrease, rise of temperature, urinary tract infection, vault granuloma in both the cases.

Results: There was no significant difference in terms of age, parity, body weight, uterine weight. The mean estimated blood loss in $\mathrm{VH}(100 \pm 32.8 \mathrm{ml})$ was lower compared to LAVH $(126.5 \pm 39.8 \mathrm{ml})$ group. Comparing the relief of post-operative pain, less analgesic was required, rapid patient recovery, and hospital stay time was less in LAVH group than VH group.

Conclusion: LAVH is clinically beneficial in terms of less post-operative pain and less quantity of analgesia used, rapid patients' recovery and shorter hospital stay than $\mathrm{VH}$. It is useful for patients who prefer laparoscopy for cosmetic purpose.

Key-words: Vaginal Hysterectomy, Laparoscopy.

\section{Introduction}

Hysterectomy is one of the commonest surgical procedures performed in gynaecological practice. Usually the abdominal route is used for this operation. But with the advancement of newer techniques, considering uterine size (less than or equal to 20 weeks), the vaginal route has also become an option. Even though there are some benefits of vaginal over abdominal hysterectomy ( $\mathrm{AH}$ ), $70-80 \%$ of all hysterectomies are performed abdominally ${ }^{1}$. Vaginal hysterectomy $(\mathrm{VH})$ is associated with lower morbidity and more rapid postoperative recovery than abdominal hysterectomy ${ }^{2-4}$. But this technique is not used frequently in patients whose choice of treatment is hysterectomy but uterine size perceived as more than 20 weeks size and concerned surgeon preferred abdominal route. Several times laparoscopic evaluation revealed that the size of the uterus was actually within 20 weeks so $\mathrm{VH}$ could have been possible in those cases and the decision of $\mathrm{AH}$ is inappropriate ${ }^{5}$.

In above-mentioned cases, Laparoscopic assisted vaginal hysterectomy (LAVH) could be performed easily instead of AH which was invented by Harry Reich in 1989 . Day by day this method has become a popular alternative to abdominal hysterectomy in cases difficult to manage via vaginal route alone ${ }^{7}$. Since then, numerous articles using the same procedure has been published and a variety of different methods have also been suggested to improve this surgical technique ${ }^{8-14}$. The previous study also indicates that LAVH is better than by $A H^{5,6}$. LAVH has become an alternative to conventional $\mathrm{AH}$ and more patients prefer the laparoscopic approach for cosmetic purpose and faster postoperative recovery rate ${ }^{15-20}$.

Performing a laparoscopy before $\mathrm{AH}$, Kovac et $\mathrm{al}^{4}$ showed that $90 \%$ of hysterectomies could be performed through vaginal route rather than abdominal one. The gynecologists who are not prepared to perform difficult vaginal procedures, LAVH may be an alternative opportunity to become familiar with the vaginal approach ${ }^{21}$. LAVH was introduced to allow surgeons with limited experience in vaginal surgery to remove the uterus without an abdominal incision in the presence of pelvic adhesion, endometriosis, adnexal disease or large uterus ${ }^{22}$. Since the invention of Laparoscopicassisted hysterectomy by Reich et al7 in 1989, there have been several modifications of the technique.

Brig Gen Liza Chowdhury, MBBS, MCPS, DGO, FCPS, Adviser Specialist \& Head, Department of Obstetrics and Gynaecology, CMH, Dhaka 
Initially, the distinction between laparoscopic hysterectomy (LH) and LAVH was based on the approach for ligation of the uterine arteries, $\mathrm{LH}$ was considered if the uterine arteries are ligated laparoscopically and LAVH if they are ligated vaginally. This study was designed to compare the efficacy and outcome of LAVH and traditional VH.

\section{Materials and Methods}

This prospective comparative study was carried out in Combined Military Hospital and Hitech Multicare Hospital, Dhaka from January 2011 to December 2013. A total of 100 non-malignant patients were selected for LAVH and VH by the same surgeon and were divided into two groups. Patients with the size of the uterus more than that of 20 weeks pregnancy, history of previous two or more cesarean sections, having cardiac or pulmonary diseases and contraindications for lithotomic position were excluded from the study. Patients outcomes measured for two groups were studied comparatively in terms of demographic and socioeconomic details; uterine weight, indication for operation, operation time, cost, estimated blood loss, post-operation pain control, hospital stay, time to return to normal activity, intra and postoperative complications, patients recovery time and histopathological findings. All statistical analyses were performed with SPSS 16.0 for Windows and $p<0.05$ was considered to be statistically significant. Student's t-test and chi-square test were used as appropriate.

\section{Results}

There was no statistically significant difference between the groups as to the mean age, parity, body weight and uterine weight (Table-I).

Table-I: Patients' profile of the study groups

\begin{tabular}{|l|c|c|}
\hline & LAVH(n=50) & VH(n=50) \\
\hline Age (Year) & $44.7 \pm 3.5$ & $42.7 \pm 5.8$ \\
\hline Parity & $2(1-3)$ & $3(2-4)$ \\
\hline Body Weight (Kg) & $59 \pm 6.0$ & $57.4 \pm 7.7$ \\
\hline Uterine Weight (g) & $273.2 \pm 126.4$ & $265.6 \pm 76.9$ \\
\hline
\end{tabular}

't' test was done, $p>0.05$

Fibroid uterus was the most common indication for both the groups (Table-II).

Table-II: Distribution of patients according to indications of Hysterectomy

\begin{tabular}{|l|c|c|}
\hline Indication & LAVH (n=50) & VH (n=50) \\
\hline Fibroid Uterus & 40 & 35 \\
\hline Endometrioses & 4 & 2 \\
\hline Pelvic Inflammatory Diseases & 2 & 3 \\
\hline Cervical Dysplasia & 1 & 3 \\
\hline Dysfunctional Uterine Bleeding & 3 & 7 \\
\hline
\end{tabular}

Operation time was slightly longer and the operation cost was higher in the LAVH group compared to VH group. The mean amount of blood loss in both groups was nearly similar.
This was verified by the postoperative by observing the operative and postoperative blood loss. Comparing the relief of post-operative pain, less Diclofenac was required for LAVH group $(70.38 \pm 13.45 \mathrm{mg})$ than $\mathrm{VH}$ group $(75.18 \pm 16.45$ $\mathrm{mg}$ ). The postoperative hospital stay and the time to return to work were nearly similar in both groups (Table-III).

Table-III: Characteristics and clinical variables of the study groups

\begin{tabular}{|l|c|c|}
\hline & LAVH (n=50) & VH (n=50) \\
\hline Operative Time (minutes) & $64.8 \pm 11.1$ & $54.8 \pm 12.3$ \\
\hline Operative Cost (Median) US\$ & 393 & 321 \\
\hline Estimated Blood loss (ml) & $126.5 \pm 39.8$ & $100 \pm 32.8$ \\
\hline Quantity of analgesics (Diclofenac mg) & $70.4 \pm 13.5$ & $75.2 \pm 16.5$ \\
\hline Hospital Stay (days) & $1.2 \pm 0.7$ & $1.5 \pm 0.7$ \\
\hline Time to return to work (weeks) & $2.4 \pm 1.1$ & $2.8 \pm 1.1$ \\
\hline
\end{tabular}

Postoperative $\mathrm{Hb}$ decrease was not significantly different between the two modalities. There is no significant difference in the two groups in the development of postoperative complications like temperature, urinary tract infection, incisional wound infection, the appearance of vault granuloma. There was no surrounding visceral injury; rehospitalization and laparotomy were not required in any of the groups (Table-IV).

Table-IV: Complication of hysterectomy of the patients among study groups

\begin{tabular}{|c|c|c|}
\hline & LAVH (n=50) & VH $(n=50)$ \\
\hline \multicolumn{3}{|l|}{ Intraoperative } \\
\hline Requiring blood Transfusion & $2 \%$ & $3 \%$ \\
\hline \multicolumn{3}{|l|}{ Postoperative } \\
\hline Hb decrease (Mean \pm SD) & $1.2 \pm 0.7$ & $1.3 \pm 0.6$ \\
\hline Febrile $\left(>38^{\circ} \mathrm{C}\right)$ & 3 & 5 \\
\hline Urinary tract infection & 3 & 3 \\
\hline Incisional Wound Infection & 0 & 0 \\
\hline Vault granuloma & 1 & 2 \\
\hline
\end{tabular}

\section{Discussion}

Early discharge (in terms of hours) from the hospital also has a major cost implication in LAVH group than that of VH. The quicker recovery in the postoperative period and the interval between surgery and return to work are two major advantages with the laparoscopic and vaginal procedure. The time of discharge from the hospital and the time to resume normal activity or complete recovery were proposed as better criteria for the patients' postoperative clinical judgment. In this study, the length of hospitalization and the mean time to resume normal activity were slightly less (in terms of days) in LAVH than that of $\mathrm{VH}$ group.

As for the estimated blood loss, there was no significant difference between LAVH and VH group which was similar to most previous studies ${ }^{11,14}$. Patients in the LAVH group needed fewer analgesics compared to those in the VH group ${ }^{10,11,13,15}$. Several studies also showed that the hospital stay was significantly shorter in LAVH group than $\mathrm{VH}$ group ${ }^{13-17}$. The operative cost for the LAVH group was slightly higher than that for $\mathrm{VH}$ group ${ }^{10,13,15,17}$ which is similar to this study. 
As demonstrated by one study, if reusable instrumentation is used and operative times made efficiently, the operative cost for the LAVH group may be reduced to less than that of the $\mathrm{VH}$ group ${ }^{18}$. Time to return to work was slightly shorter in LAVH group and VH group as in other studies ${ }^{19,20}$ which is similar to this study. Nevertheless, multiple myoma and large uterus often are barriers. So,an accurate preoperative diagnosis is essential. However, in this study, none of the cases from both groupswas converted to $\mathrm{AH}$ and the incidence of complications was low.

Data from this study justify that, LAVH is clinically beneficial in terms of less postoperative pain. Thus, in patients with no need for abdominal surgery (e.g. no significant adhesions or endometriosis), vaginal hysterectomy seems a better and faster approach than $\mathrm{AH}^{23}$. The patients were quite similar in two groups and group assignment was not based on patient's selection of surgical procedure or on physician's selection of particular patients, it was based on the assignment of attending surgeon. As both procedures were performed by the same surgeon, the skill remains unquestionable. Based on the results of this study, the following conclusion can be drawn: First, many patients traditionally treated with $\mathrm{AH}$ may be treated via VH or LAVH. Second, surgical time for LAVH was slightly greater than that for $\mathrm{VH}$. (Technologic improvements, such as endoscopic stapler-cultures, may decrease the difference.) Third, postoperative pain was less with LAVH than that of VH. Fourth, hospital stay was shorter with LAVH than with $\mathrm{VH}$ in terms of hours. If $\mathrm{VH}$ and $\mathrm{LAVH}$ could be done as an outpatient basis then $\mathrm{VH}$ and LAVH both might be economically beneficial for the patients. Finally, LAVH has no consequential cost benefit over $\mathrm{VH}$ when only hospital costs are considered.

\section{Conclusion}

$\mathrm{LAVH}$ requires less post-operative analgesia than $\mathrm{VH}$. With technological improvement and expertise, the duration of operation and hospital stay can also be minimized. It is safe to say that,in cases where hysterectomy is the treatment of choice and $\mathrm{VH}$ is contraindicated, to avoid the abdominal route, surgeons can easily go for LAVH if the centre is properly equipped. So, the gynaecologists should be encouraged to familiarize themselves with LAVH technique which may become time-demanding in days to come.

\section{References}

1. Harry R, Lisa R. Laparoscopic hysterectomy in current gynecological practice. Gynocol and perinatal prac 2003; 3(1):32-40.

2. Kaunitz AM, Meredith $S$, Inki $P$ et al. Levonorgestrel-releasing intrauterine system and endometrial ablation in heavy menstrual bleeding: A systematic review and meta-analysis. Obstet Gynecol 2009; 113(5):1104-16.
3. Cheng YL, Jia HF, Wei CC et al. Comparison of total laparoscopic Hysterectomy and Laparoscopic Assisted Vaginal Hysterectomy, Gynecological and Obstetric Investigation 2002; 53:214-9.

4. Nezhat $C$, Winer WK, Nezhat $F$ et al. Video laparoscopy and Video laseroscopy-Alternative to surgery? Female Patient 1988; 13:46.

5. Kristen AM. Factors associated with increased charges for hysterectomy, Obstetrics and gynecology 2006; 107:1057-63.

6. Erin MH. Total laparoscopic hysterectomy in obese versus non obese patients. Obstetrics \& Gynecology 2004; 103:674-80.

7. Wood C, Maher P, Hill D. Replacement of abdominal hysterectomy by the laparovaginal technique-its success and limitations. Aust NZJ Obstet Gynaecol 1994; 34:571-4.

8. Olsson $\mathrm{JH}$, Ellstrom M, Hahlin M. A randomized prospective trial comparing laparoscopic and abdominal hysterectomy. $\mathrm{Br} \mathrm{J}$ Obstet Gynaecol 1996; 103:345-50.

9. Garry R, Fountain J, Mason S et al. The evaluate study: two parallel randomised trials, one comparing laparoscopic with abdominal hysterectomy, the other comparing laparoscopic with vaginal hysterectomy. BMJ 2004: 328:129-33

10. Farquhar CM, Steiner CA. Hysterectomy rates in the United States 1990-1997. Obstet Gynecol 2002; 99(2):229-34

11. Lumsden MA, Twaddle $S$, Hawthorn $R$ et al. A randomised comparison and economic evaluation of laparoscopic-assisted hysterectomy and abdominal hysterectomy. Br J Obstet Gynaecol 2000; 107(11):1386-91.

12. Lee CL, Soong YK. Laparoscopic Hyterectomy: Is dissecting the ureter necessary? Int Surg 1995; 80:167-9.

13. Richard SR, Sompkins S. Laparoscopic supracervical hysterectomy versus laparoscopic-assisted vaginal hysterectomy. J Am Assoc Gynecol Laparosc 1995; 2:431-5.

14. McPherson K, Metcalfe MA, Herbert Aet al. Severe complications of hysterectomy: The value study. Br J Obstet Gynaecol 2004; 111(7):688-94.

15. Hyuk Jung, Ju-Seong Lim, Hye-Jin Cho. Comparison of bipolar coagulator and ultrasonic surgical system on lapascopic assisted vaginal hysterectomy. Journal of Gynecologic Surgery 2005; $21(2): 73-9$.

16. AK Trehan. Hysterectomy: Towards an overnight stay. Gynaecologic Endoscopy 2002; 11(4):181-7.

17. Boukerrou M, Lambaudie E, Narducci Fet al. Hysterectomy for benign lesions. What is left for the abdominal route? JGynecol Obstet Biol Reprod (Paris) 2001; 30(6):584-9.

18. Clayton RD, Hawe J, Garry R. Laparoscopically assisted hysterectomy for the large uterus. Gynaecologic Endoscopy 1999; 8(4):219-23.

19. Mehra S, Bhat V, Mehra G. Laparoscopic vs abdominal vs vaginal hysterectomy. Gynaecologic Endoscopy 1999; 8(1):29-34.

20. Kim DH, Chi JS. Total Laparoscopic Hysterectomy with suture technique. Gynaecologic Endoscopy 1998; 7(5):259-65.

21. Varol N, Healey M, Tang $P$ et al. Ten yearreviews of hysterectomy morbidity and mortality. Can we change direction? Aust NZJ Obstet Gynaecol 2001; 41:295-302.

22. Katherine A. Total laprascopy hysterectomy vs Total abdominal hysterectomy: Cohort Review of Patients with Uterine Neoplasia. JSLS 2005; 9:277-86.

23. Jacobson MT, Osias J, Bizhang R et al. The direct trocar technique: An alternative approach to abdominal entry for laparoscopy. JSLS 2002; 6(2):169-74. 